\title{
Evaluation of Wavelength-Dependent Detection Efficiency of Neutron-Sensitive Microchannel Plate Detector
}

\author{
Kenichi Watanabe, ${ }^{*}$ Triestino Minniti, ${ }^{1}$ Winfried Kockelmann, ${ }^{1}$ \\ Robert Dalgliesh, ${ }^{1}$ Genoveva Burca, ${ }^{1}$ and Anton S. Tremsin ${ }^{2}$ \\ Graduate School of Engineering, Nagoya University, Furo, Chikusa, Nagoya, Aichi 464-8603, Japan \\ ${ }^{1}$ STFC, Rutherford Appleton Laboratory, ISIS Facility, Harwell, OX11 0QX, United Kingdom \\ ${ }^{2}$ Space Science Laboratory, University of California at Berkeley, CA 94720 Berkeley, U.S.A.
}

(Received April 7, 2017; accepted August 21, 2017)

Keywords: microchannel plate, neutron imaging detector, time-of-flight energy analysis, detection efficiency, detector activation

The microchannel plate (MCP)/Timepix is one of the promising candidates for highperformance neutron imaging detectors. Since it is important to know the detection efficiency to plan experiments, we propose simple procedures to evaluate the wavelength-dependent detection efficiency of the MCP/Timepix detector. The wavelength-dependent efficiency curve can easily be evaluated by comparison with the neutron-induced prompt gamma-ray counts. The efficiency increases with the wavelength up to roughly $5 \AA$ and eventually saturates. All neutrons with a wavelength of more than $5 \AA$ are fully absorbed in the neutron-sensitive MCP. The activation effect in the MCP/Timepix detector is also investigated. We confirmed additional counts originating from the activation effect of aluminum, which was used in the detector entrance window. The contribution of the additional counts due to the detector activation was less than $10^{-4}$ compared with the neutron counts.

\section{Introduction}

Energy-resolved neutron imaging has been expected to expand the applicability of neutron analysis methods in materials science. Energy-resolved images can effectively be performed by combining a pulsed neutron source and a time-resolving camera in the neutron time-offlight (TOF) method. Pulsed-neutron-source facilities have been built or are being prepared to fabricate instruments for energy-selective neutron imaging, such as RADEN in the Japan Proton Accelerator Research Complex (J-PARC) and Imaging and Materials Science and Engineering (IMAT) in ISIS, which is under commission at the present time. ${ }^{(1-3)}$ To fully utilize the capabilities of pulsed sources in imaging experiments, novel detection technologies are being developed. These detectors should have a time-resolving capability, high detection efficiency, high spatial resolution, and stability. At present, a promising candidate is the borated-microchannel plate (MCP) detector combined with an array of Timepix chips, which are high-performance applicationspecific integrated circuit (ASIC) readout chips. ${ }^{(4,5)}$ The borated-MCP, which is fabricated by Nova Scientific Inc., can work as a highly efficient neutron-to-electron converter with high spatial resolution. A stack of two or three MCPs can be used to perform sufficient amplification of the

*Corresponding author: e-mail: k-watanabe@energy.nagoya-u.ac.jp

http://dx.doi.org/10.18494/SAM.2017.1625

ISSN 0914-4935 C MYU K.K. 
electron signal produced by the neutron-sensitive MCP. The electron signal is read out by a finely pixelated Timepix readout chip with $55 \mathrm{~mm}$ pixel size and a $256 \times 256$ matrix of pixels per single chip with a $14 \times 14 \mathrm{~mm}^{2}$ active area. The Timepix chip can also be operated in the time-resolved counting mode. This type of detector is being installed at the ISIS pulsed neutron source as the time-resolving neutron imaging camera. The most important advantage of this detector is its small pixel size. At present, the $55 \mu \mathrm{m}$ pixel size is the smallest one in neutron imaging detectors and provides the highest spatial resolution. In addition, the small pixel size of the Timepix chip can reduce count rates per pixel and allows operation under high flux conditions. ${ }^{(6,7)}$

Some basic performances of the MCP/Timepix detector, such as the detection efficiency, and spatial and time resolutions, have already been investigated. ${ }^{(8,9)}$ However, no detailed study on the energy-dependent or wavelength-dependent detection efficiency has been carried out for the present generation of devices, except for the theoretical predictions ${ }^{(4,10)}$ and experimental data are sparse. ${ }^{(8)}$ This information is useful for planning experiments of new energy-selective imaging applications, such as neutron resonance transmission imaging and stress mapping via Bragg-edge analysis. ${ }^{(11,12)}$ In this paper, we propose simple procedures for evaluating the energy- or wavelength-dependent detection efficiency of the MCP/Timepix detector. In addition, we investigate the effects related to detector activation by neutrons. This contribution to the detector response is also important when an opaque sample with very low neutron transmittance is measured. Activation signals might have a relatively significant contribution in that case.

\section{Experimental Setup}

The neutron-sensitive MCP has a relatively high content of boron in the glass matrix, in which boron acts as a converter of slow neutrons to energetic charged particles through ${ }^{10} \mathrm{~B}(\mathrm{n}, \alpha){ }^{7} \mathrm{Li}$ reactions. In addition, gadolinium atoms are also doped into the glass and convert neutrons to electrons emitted by internal conversion after Gd neutron absorption. These energetic particles induce the emission of secondary electrons from the inner surface of MCP pores. The emitted electrons are multiplied in the MCP and read out with the Timepix chip, which was developed within the Medipix collaboration at CERN. ${ }^{(13)}$ The detector used in these experiments utilizes a chevron stack of two MCPs. The top MCP is neutron sensitive, $50 \mathrm{~mm}$ in diameter, and $0.8 \mathrm{~mm}$ thick and has $8 \mu \mathrm{m}$ pores hexagonally packed with $10.5 \mu \mathrm{m}$ pitch. The bottom MCP is a standard electron multiplier MCP manufactured by Hamamatsu Photonics. The standard MCP is $50 \mathrm{~mm}$ in diameter and $0.6 \mathrm{~mm}$ thick and has $10 \mu \mathrm{m}$ pores with $12 \mu \mathrm{m}$ pitch. The MCP stack is mounted at roughly $0.5 \mathrm{~mm}$ from the Timepix readout chip. ${ }^{(5)}$ The present MCP/Timepix detector uses a $2 \times 2$ array of Timepix chips, forming a matrix of $512 \times 512$ pixels. The total field of view is 28 $\times 28 \mathrm{~mm}^{2}$. The Timepix chip was operated with the event timing mode in order to acquire timeresolved images. In neutron TOF measurements, energy-resolved neutron images can be obtained. In the event timing mode, the time resolution is tunable depending on the recorded time range in TOF measurements. The minimum time resolution is $10 \mathrm{~ns}$ but, in this case, the recorded TOF range will be quite narrow owing to the finite number of recordable time bins. ${ }^{(5)}$ Generally, the energy or wavelength resolution depends on not only the detector time resolution but also the neutron pulse width of the beamline.

Almost all experiments were conducted on the Larmor beamline in ISIS. The Larmor beamline is one of the beamlines of ISIS Target Station 2 (TS2), which is fundamentally a multipurpose 
instrument for small-angle neutron scattering (SANS), diffraction, and spectroscopy utilizing the Larmor precession of polarized neutrons. This beamline is operated with the repetition rate of 10 $\mathrm{Hz}$, a maximum neutron intensity around $3 \AA$ in wavelength, and a $30 \times 30 \mathrm{~mm}^{2}$ beam size. In these tests, the flight path from the neutron source to the detector was roughly $26 \mathrm{~m}$.

To evaluate the energy- or wavelength-dependent neutron detection efficiency, we must obtain the TOF spectrum of the incident neutron beam. The spectrum shape can be determined by measuring prompt gamma rays emitted from a neutron shielding plate, which can fully absorb relatively low-energy neutrons. Since an energy spectrum of prompt gamma rays is independent of incident neutron energy, the detector response to the prompt gamma ray can be used as an appropriate index of incident neutron intensity. A 3-mm-thick Cd plate was used for the neutron shielding. We placed the plate in front of the MCP detector entrance window when measuring the prompt gamma rays. In order to evaluate the influence of stray background neutrons, we also measured the detector response when the plate was placed at a distance of $350 \mathrm{~mm}$ from the detector.

We additionally confirmed the absolute detection efficiency by comparison with a reference detector, whose efficiency was already determined. This experiment was conducted at the IMAT beamline in ISIS, which is a neutron imaging beamline in TS2. ${ }^{(2,3)}$ The IMAT has almost the same neutron energy spectrum as the Larmor beamline because both beamlines view the same neutron moderator. The flight path of the IMAT beamline is roughly $56 \mathrm{~m}$. The neutron flux of the IMAT beamline was evaluated with the reference detector. In the IMAT beamline, a small GS1 Li glass scintillator, in which $\mathrm{Li}$ is in natural isotopic abundance, was used as reference detector. Neutrons are almost transparent to this detector because of its small size and low ${ }^{6} \mathrm{Li}$ content. This means that the detection efficiency is simply proportional to the neutron absorption cross section of ${ }^{6} \mathrm{Li}$, which is proportional to the neutron wavelength or inversely proportional to the neutron velocity, and can easily be corrected.

In order to check the detector activation effect, we irradiated the detector for more than $4 \mathrm{~h}$ at the Larmor beamline. Just after stopping neutron irradiation, we began to measure the detector counts due to its activation.

\section{Results and Discussion}

Figure 1 shows the TOF spectrum of neutron counts obtained with the MCP/Timepix detector at the Larmor beamline. The counts of the MCP/Timepix detector are influenced by the overlap counting loss. All the results shown in this paper were corrected with the overlap correction procedures proposed by Tremsin et al. ${ }^{(14)}$ Neutron pulses have maximum intensity at around 16 ms, which corresponds to the neutron wavelength of $2.5 \AA$. Figure 2 shows the TOF spectra of the neutron-induced prompt gamma ray from the $\mathrm{Cd}$ shielding plate placed at different distances from the MCP detector. The prompt gamma-ray spectra also have a peak shape similar to that of neutron pulses. This means that the MCP/Timepix detector has nonzero sensitivity to the prompt gamma rays. As described above, the energy spectrum of the prompt gamma ray is independent of the incident neutron energy and the prompt gamma-ray detection efficiency is constant. This means that the prompt gamma-ray counts are proportional to the incident neutron intensity if all neutrons are absorbed by the shielding plate. In a 3-mm-thick Cd plate, almost all neutrons are absorbed at wavelengths greater than $0.5 \AA$. In this wavelength region, the relative neutron 


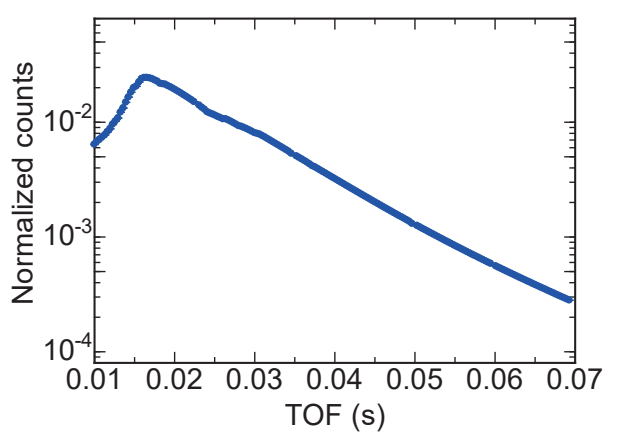

Fig. 1. (Color online) TOF spectrum of neutron counts obtained with the MCP/Timepix detector at the Larmor beamline.

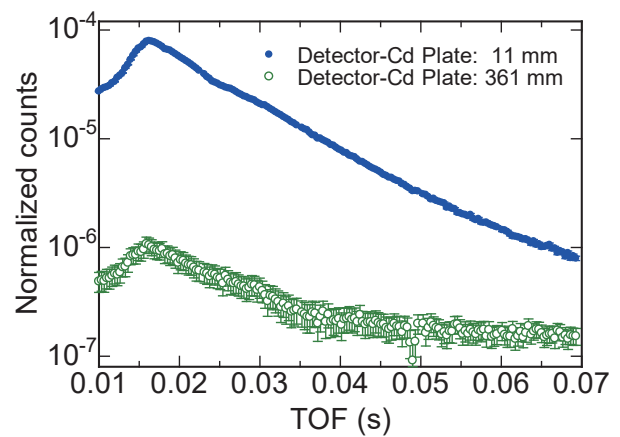

Fig. 2. (Color online) TOF spectra of neutroninduced prompt gamma ray from $\mathrm{Cd}(t=3 \mathrm{~mm})$ shielding plate. This spectrum was obtained at the Larmor beamline.

detection efficiency can be evaluated from the ratio of the neutron counts to the prompt gamma-ray counts. Figure 3 shows the wavelength dependence of the relative neutron detection efficiency of the $\mathrm{MCP} /$ Timepix detector obtained by comparison with the results for the $\mathrm{Cd}$ prompt gamma rays. The calculated absorption with ${ }^{10} \mathrm{~B}$ in the MCP is also plotted. In this calculation, the ${ }^{10} \mathrm{~B}$ content was assumed to be $2.3 \times 10^{20}$ atoms $/ \mathrm{cm}^{2}$. Although we have no details on the composition of the neutron-sensitive MCP, this ${ }^{10} \mathrm{~B}$ content is roughly consistent with the description in the patent of Nova Scientific Inc. ${ }^{(15)}$ The MCP/Timepix detector can fully stop neutrons with a wavelength of more than $5 \AA$. We note that the full stoppage of neutrons does not mean $100 \%$ detection. Some fraction of neutron absorption events cannot induce a significant level of signals and might be lost as uncounted events. The counting efficiency depends on the MCP gain and the threshold level of the Timepix readout chip. In these experiments, the MCP gain was not fully optimized and some events fell below the threshold level of the discriminator. By increasing the applied high voltage to the MCP or adjusting the threshold level, the absolute detection efficiency would be improved to the detection efficiency values exceeding $50 \%$, as demonstrated previously. ${ }^{(4,8,10)}$

In order to validate the simple evaluation procedures of the wavelength-dependent detection efficiency, we compared the efficiency curve with the absolute detection efficiency measured at the IMAT beamline, where the neutron flux was evaluated with the GS1 reference detector. Figure 4 shows the absolute detection efficiency of the MCP/Timepix detector evaluated at the IMAT beamline. The efficiency curve simply evaluated with the prompt gamma ray agrees with the absolute efficiency obtained by the GS1 reference detector. The proposed simple procedure can evaluate the wavelength-dependent detection efficiency without the reference detector, whose detection efficiency curve has already been determined.

Figure 5 shows the time trend of the detector counts before and after closing the neutron shutter. The neutron shutter was closed at the vertical line in this figure. The detector was still counting with a low count rate even after closing the neutron shutter. The count rate immediately decreased without the neutron beam. In order to characterize the decay behavior, the short measurements were repeatedly conducted. Figure 6 shows the decay characteristics of the count rate just after closing the neutron shutter. Since the count rate exponentially decreased, the data were fitted with an exponential decay curve with a constant background. The half-life determined by the fitting process is $155 \mathrm{~s}$. This decay behavior is considered to originate from activated ${ }^{28} \mathrm{Al}$, which has a half-life of $2.24 \mathrm{~min}$. The detector entrance window consists of a sub-millimeter-thick Al plate at 


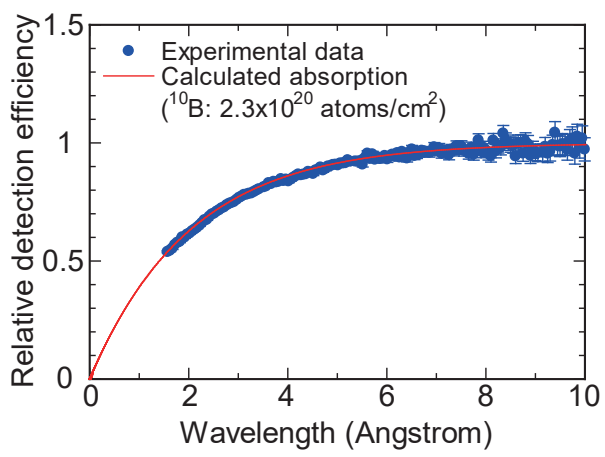

Fig. 3. (Color online) Wavelength dependence of relative neutron detection efficiency of the MCP/ Timepix detector obtained by comparison with the results for $\mathrm{Cd}$ prompt gamma rays. Calculated absorption with ${ }^{10} \mathrm{~B}\left({ }^{10} \mathrm{~B}\right.$ content: $2.3 \times 10^{20}$ atoms/ $\mathrm{cm}^{2}$ ) is also plotted.

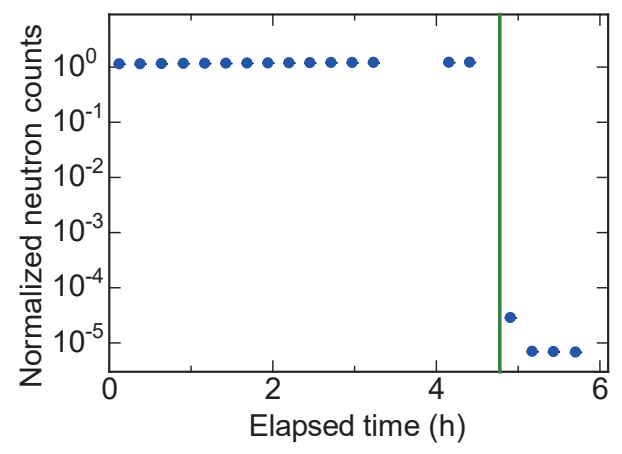

Fig. 5. (Color online) Time trend of detector counts before and after closing the neutron shutter.

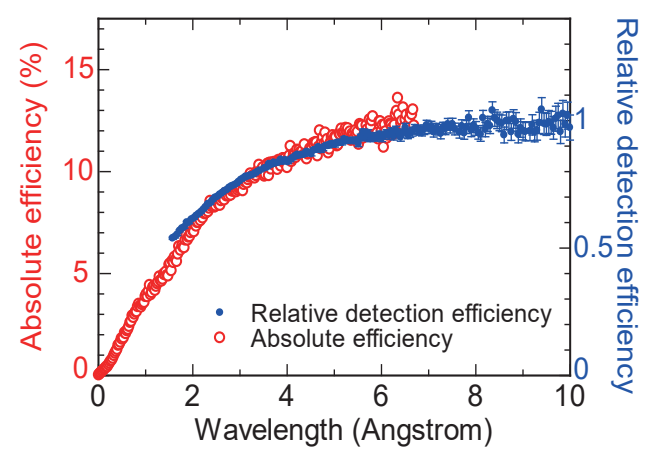

Fig. 4. (Color online) Absolute detection efficiency of the MCP/Timepix detector evaluated at the IMAT beamline.

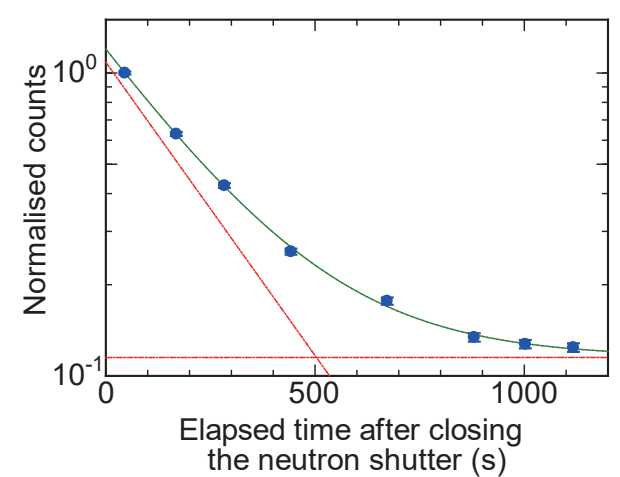

Fig. 6. (Color online) Decay characteristics of count rate owing to detector activation. This time trend started to be recorded just after closing the neutron shutter.

the active area. Beta particles emitted from the activated entrance window can reach the MCP and generate signals. After neutron irradiation for half an hour, the aluminum activation completely saturates because of its short half-life. The contribution of additional counts due to the activation is less than $10^{-4}$ compared with neutron counts.

\section{Conclusions}

We proposed simple procedures to evaluate the wavelength-dependent detection efficiency of the MCP/Timepix detector. We evaluated the wavelength-dependent efficiency curve compared with the neutron-induced prompt gamma-ray counts. The efficiency increased with increasing wavelength and eventually saturated at wavelengths above $5 \AA$. All neutrons with wavelength longer than $5 \AA$ were fully absorbed. This efficiency behavior was consistent with the boron content in the MCP glass matrix. 
We additionally investigated the activation effect in the MCP/Timepix detector. We confirmed that additional counts originate from the neutron activation of aluminum, which was used as the detector entrance window. The contribution of the additional counts due to detector activation was less than $10^{-4}$ compared with the neutron counts. This contribution was quite small but should be considered when opaque samples with low neutron transmittance are measured.

\section{Acknowledgments}

This work was partially supported by the JSPS Program for Advancing Strategic International Networks to Accelerate the Circulation of Talented Researchers.

\section{References}

1 T. Shinohara and T. Kai: Neutron News 26 (2015) 11.

2 T. Minniti, W. Kockelmann, G. Burca, J. F. Kelleher, S. Kabra, S. Y. Zhang, D. E. Pooley, E. M. Schooneveld, Q. Mutamba, J. Sykora, N. J. Rhodes, F. M. Pouzols, J. B. Nightingale, F. Aliotta, L. M. Bonaccorsi, R. Ponterio, G. Salvato, S. Trusso, C. Vasi, A. S. Tremsin, and G. Gorini: J. Instrum. 11 (2016) C03014.

3 W. Kockelmann, G. Frei, E. H. Lehmann, P. Vontobel, and J. R. Santisteban: Nucl. Instrum. Methods Phys. Res., Sect A 578 (2007) 421.

4 A. S. Tremsin, V. Dangendorf, K. Tittelmeier, B. Schillinger, M. Schulz, M. Lerche, and W. B. Feller: J. Instrum. 10 (2015) P07008.

5 A. S. Tremsin, J. V. Vallerga, J. B. McPhate, and O. H. W. Siegmund: Nucl. Instrum. Methods Phys. Res., Sect. A 787 (2015) 20.

6 A. S. Tremsin, M. Morgano, T. Panzner, E. Lehmann, U. Filgers, J. V. Vallerga, J. B. McPhate, O. H. W. Siegmund, and W. B. Feller: Nucl. Instrum. Methods Phys. Res., Sect. A 784 (2015) 486.

7 A. S. Tremsin, J. B. McPhate, J. V. Vallerga, O. H. W. Siegmund, J. S. Hull, W. B. Feller, and E. Lehmann: Nucl. Instrum. Methods Phys. Res., Sect. A 605 (2009) 103.

8 A. S. Tremsin, J. B. McPhate, J. V. Vallerga, O. H. W. Siegmund, J. S. Hull, W. B. Feller, and E. Lehmann: Nucl. Instrum. Methods Phys. Res., Sect. A 604 (2009) 140.

9 A. S. Tremsin, J. B. McPhate, J. V. Vallerga, O. H. W. Siegmund, W. B. Feller, E. Lehmann, and M. Dawson: Nucl. Instrum. Methods Phys. Res., Sect. A 628 (2011) 415.

10 A. S. Tremsin, W. B. Feller, R. G. Downing, and D. F. R. Mildner: IEEE Trans. Nucl. Sci. 52 (2005) 1739.

11 A. S. Tremsin, J. B. McPhate, J. V. Vallerga, O. H. W. Siegmund, W. Kockelmann, E. M. Schooneveld, N. J. Rhodes, and W. B. Feller: IEEE Trans. Nucl. Sci. 59 (2012) 3272.

12 A. S. Tremsin, J. B. McPhate, J. V. Vallerga, O. H. W. Siegmund, W. Kockelmann, A. Steuwer, and W. B. Feller: IEEE Sens. J. 11 (2011) 3433.

13 X. Llopart, R. Ballabriga, M. Campbell, L. Tlustos, and W. Wong: Nucl. Instrum. Methods Phys. Res., Sect A 581 (2007) 485.

14 A. S. Tremsin, J. V. Vallerga, J. B. McPhate, and O. H. W. Siegmund: J. Instrum. 9 (2014) C05026.

15 W. B. Feller, P. L. White, and W. J. S. Zhong: Neutron detection and collimation, US Patent \#9,082,907, Nova Scientific Inc. (2015).

\section{About the Authors}

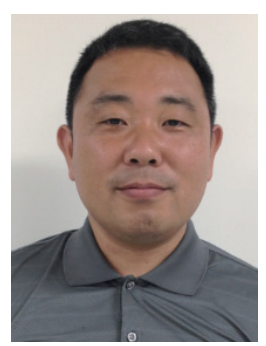

Kenichi Watanabe received his M.S. and Ph.D. degrees from Nagoya University, Japan, in 1998 and 2003, respectively. From 2000 to 2007, he was a research assistant at Nagoya University, Japan. Since 2007, he has been an associate professor at Nagoya University. His research interests are in radiation detectors. 


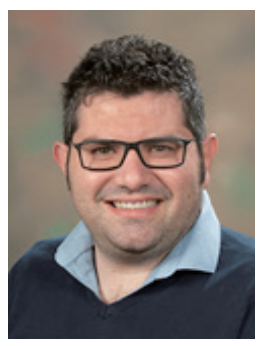

Triestino Minniti received his M.S. degree from Messina University, Italy, in 2009 and Ph.D. degree from Messina University and the National Institute of Nuclear Physics (INFN), Italy, in 2012. From 2012 to 2014, he was a Postdoctoral Researcher at INFN-LNS and from 2014 to 2016 at the Science and Technology Facility Council, Rutherford Appleton Laboratory, ISIS facility, United Kingdom. Since 2016, he has been Scientist at the Science \& Technology Facility Council, Rutherford Appleton Laboratory, ISIS facility, United Kingdom. His research interests are in neutron imaging techniques.

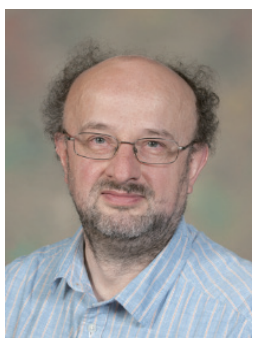

Winfried Kockelmann is a physicist and received his Ph.D. degree from the University of Bonn, Germany, in 1995. From 1995, he worked at the ISIS pulsed neutron source at the Rutherford Appleton Laboratory in the UK, initially on assignment from Bonn University and Forschungszentrum Jülich, Germany, then as a member of the ISIS Crystallography Group. He supports the materials science programme of ISIS and is actively involved in instrument development projects. His scientific interests include phase transitions, structural magnetism, and the development of neutron techniques for the analysis of cultural heritage objects.

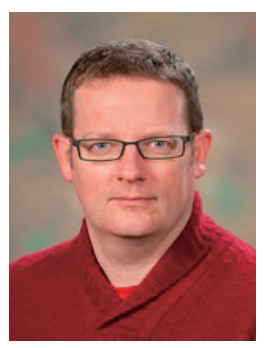

Robert Dalgliesh received his Ph.D. in physics from the University of Bristol, UK. He has worked at the ISIS pulsed neutron source at the Rutherford Appleton Laboratory since 2000 as a member of the Large Scale Structures Group working on both reflectometers and small angle scattering instruments while also collaborating in the development of novel neutron spin-echo techniques. His scientific interests include the application of neutron scattering to the study of hierarchical structures and the development of novel instrumentation.

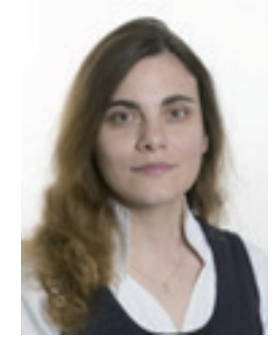

Genoveva Burca received her Ph.D. degree from The Open University, UK in materials engineering with a thesis on combining imaging and diffraction instrumentation and experimentation at the neutron spallation source of ISIS, UK. Since July 2015, she has been instrument scientist for the neutron imaging and diffraction instrument IMAT from Rutherford Appleton Laboratory, UK. Her main research interests are in neutron imaging and diffraction techniques and their applications to engineering and life sciences.

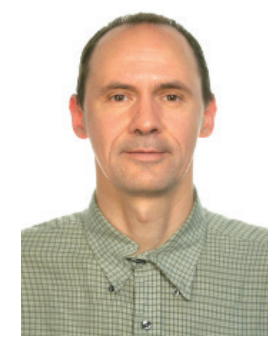

Anton S. Tremsin received his M.S. degree from the Moscow Institute of Physics and Technology in 1989 and Ph.D. degree in Applied Physics in 1992 from the Russian Academy of Sciences. From 1994 to 1996, he was an Honorary Royal Society Postdoctoral Fellow at the University of Leicester, UK and after that, he joined the Space Sciences Laboratory at the University of California at Berkeley where he is a full-time researcher at present. His research interests are in the development of novel nondestructive testing techniques and data analysis tools as well as instrumentation for high resolution photon, neutron, electron and ion detection in various applications. 